Authors’ Accepted Manuscript version, published in:

Journal:Journal of documentation

ISSN:0022-0418

Date: $12 / 2019$

Volume: ahead-of-print Issue: ahead-of-print

DOI:10.1108/JD-05-2019-0078

https://www.emerald.com/insight/content/doi/10.1108/JD-05-2019-0078/full/html

\title{
Playful interfaces to the archive and the embodied experience of data
}

Rachel Hendery (Western Sydney University) | r.hendery@westernsydney.edu.au

Andrew Burrell (University of Technology, Sydney) | andrew.burrell@uts.edu.au

\section{Abstract \\ Purpose}

This paper demonstrates the possibility for the Galleries, Libraries, Archives and Museums sector to employ playful, immersive discovery interfaces for their collections and raises awareness of some of the considerations that go into the decision to use such technology and the creation of the interfaces.

\section{Design/methodology/approach}

This is a case study approach using the methodology of research through design. The paper introduces two examples of immersive interfaces to archival data created by the authors, using these as a springboard for discussing the different kinds of embodied experiences that users have with different kinds of immersion, for example the exploration of the archive on a flat screen, a data 'cave’ or arena, or virtual reality.

\section{Findings}

The role of such interfaces in communicating with the audience of an archive is considered, for example in allowing users to detect structure in data, particularly in understanding the role of 
geographic or other spatial elements in a collection, and in shifting the locus of knowledge production from individual to community.

It is argued that these different experiences draw on different metaphors in terms of users' prior experience with more well known technologies, for example 'a performance' versus 'a tool' versus ‘a background to a conversation'.

\section{Originality/value}

The two example interfaces discussed here are original creations by the authors of this paper. They are the first uses of mixed reality for interfacing with the archives in question. One is the first mixed reality interface to an audio archive. The discussion has implications for the future of interfaces to galleries, archives, libraries and museums more generally.

Keywords: virtual reality, interfaces, archives, libraries, digital repositories, discovery, data visualisation, data visualization, linguistics, languages, mixed reality

\section{Introduction}

Librarians and archivists have long recognised that different interfaces are needed for the diverse kinds of audiences that engage with an archive (Miller, 1942, Stevens, 1950, Tagliacozzo and Kochen, 1970, Bates, 1977, Efthimiadis, 1990). This paper describes how immersive technologies such as mixed reality, together with ubiquitous computing, open up new possibilities for creating such interfaces, and discusses two examples of playful, immersive archival interfaces the authors have created. An example of the differing needs and expectations of different users can be seen by comparing scholars conducting a targeted study to users who want a 'big picture' overview of an archive's holdings. Researchers trying to answer a specific research question need a complex, flexible search interface, or a way to export the data they need 
into a form they can input into their preferred software. They will generally be willing to dig around in a database or catalogue to find exactly what they need, even if it is not apparent on first glance, or requires several hours of their time. Users who are simply curious about the holdings of the archive, on the other hand, will have less patience for complex interfaces or hidden information. Users on a mobile device or with low bandwidth will not want to have to download large files in order to explore, for example, audio or video holdings. Users less familiar with the content of the archive may not be interested in searching, sorting or filtering by key terms that may, on the other hand, be essential to domain experts. These different needs mean that multiple different 'ways in' to the archive's holding may be necessary for the different stakeholder communities.

Moving on from traditional interfaces, the rise of virtual reality (VR) and other mixed reality devices as affordable, ubiquitous platforms has opened up new possibilities for interactive, experimental interfaces to archival data. An interface no longer has to be something a user sits at a computer to access. Rather, interfaces can break out of the computer into the world around us, and users can enter the world of the computer and become part of the interface. Objects in the physical world can become markers or sites of interaction; natural movements or user actions such as gestures, or stepping, can be picked up by cameras, infrared monitoring devices, or pressure plates in a designated space; even a user's physical location in geographical space can be used to trigger relevant information in textual, audio and visual form. With the aid of a VR headset or a purpose-built space, users can step into a virtual world where everything they interact with is computer generated. The possibilities for different kinds of archive interfaces are therefore no longer limited to two-dimensional database frontends to be navigated with keyboard and mouse. 
This paper discusses two case studies of VR visualisations of archival (linguistic) data as a springboard for discussing the affordances of different immersive technologies in this space. ${ }^{1}$ The potential of a variety of different technologies to access virtual environments for visualising archival data is compared; the design and implementation process for the virtual environments as immersive data spaces are described; and conclusions are drawn about how such interfaces to an archive function as both technologies of information organisation, and embodied physical experiences. The discussion of the two quite different case studies and their realisations across a number of different kinds of platforms is intended to showcase a wide range of possibilities for such immersive interfaces, and to demonstrate the need for any archive considering implementing mixed reality approaches to adopt design principles, kinds of visualisations, and platforms that best suit their goals, audiences, and the resources they have available.

\section{Design framework}

The use of virtual interfaces to a library or archive is something that has long been anticipated. Poulter (1993) describes a virtual reproduction of library or archive shelves for users to digitally browse among. Some libraries have even created such virtual spaces in platforms such as Second Life (see Chow et al., 2010, Riha, 2010). More recently, it has become relatively common for archives to experiment with laying out documents and images before a user in a virtual space (see Lugmayr et al 2016 or the numerous examples described in Whitelaw 2015). In all of these cases, however, the intent appears to be to connect the user to a simulated archive experience, either through virtual shelves and stacks, or by simultaneously displaying large

${ }^{1}$ Some of our work discussed here has received funding from the Australian Research Council (DP180100893) 'Waves of Words: Mapping and Modelling Australia's Pacific Ties' and from the Centre of Excellence for the Dynamics of Language (Transdisciplinary and Innovation Grant). 
collections of primarily visual and textual materials on a kind of virtual table or wall. An alternative approach would be to background the archive’s mediating role, and attempt to connect the user more directly with the data in an experience that more closely approximates either the environment from which the data originate, or the research environment in which the data are typically analysed. These are the approaches taken in the case studies discussed in this paper. This is particularly effective in the case where the archival holdings, as in one of these case studies, consist of audio recordings rather than visual or textual information. The example described here is potentially the first VR experience to use spatialized audio as an interface for information discovery and retrieval.

This approach is situated in a theoretical framework from the 'first wave' of mixed reality. In the development of thinking about VR interface design through the 1990s to the present day, there has been a shift from what Laurel et al (1994) called the 'no interface' paradigm, to an approach that draws heavily on two-dimensional interfaces that users are already familiar with from their desktop computer experiences. The latter is unsurprising, as development of new media technologies have always leaned heavily on those that have gone before. Just as McLuhan (1964) claims that the 'content' of writing is speech, and the content of a film is a novel, play or opera, a natural way to think about a virtual reality experience is as a three-dimensional container for the kinds of two-dimensional content usually presented on screen-based new media, whether that be video, an animated game, or a data visualisation.

The early pioneers of virtual reality design demonstrated, however, that it does not have to be this way, suggesting that a virtual experience could be the experience rather than a simulation of an experience; that it could grant the user strange powers and ways to transform and expand their 
embodied awareness (see e.g. Lanier 1989, p 110; Laurel 1993; Laurel et al 1994; Davies 1998; Krueger quoted in Turner 2002; Heim 1993, p 128.)

Laurel et al. (1994) describe the 'no interface' approach as a 'desire to maximize naturalness, to enable the body to act directly in the world, and to minimize distraction and cognitive load'. This is the framework within which the projects described in this paper should be understood: rather than putting existing archival interfaces or tools into three-dimensional spaces, these projects connect the user more immediately to the data held by the archive. In some versions of the projects (with the HTC Vive), the user can even directly interact with that data using his or her body.

\section{Description of the visualisations}

The two visualisations discussed here are intended as a 3D exploration of linguistic time and space, using data from two different major linguistic archives. The first is a geographicallyanchored walkthrough of the data held in the Pacific and Regional Archive for Digital Sources in Endangered Cultures (PARADISEC). PARADISEC is a digital archive holding data primarily generated from linguistic fieldwork in the Pacific, and more recently, other small languages around the world (Thieberger and Barwick, 2012). It contains audio, video, and text data, some of which has been digitised and some of which was born digital. The text data takes the form of research notebooks, word lists, and even draft manuscripts of publications.

A secondary data source that is used in this visualisation is the Open Languages Archives Community (OLAC) resources. OLAC is "an international partnership of institutions and individuals who are creating a worldwide virtual library of language resources by: (i) developing consensus on best current practice for the digital archiving of language resources, and (ii) developing a network of interoperating repositories and services for housing and accessing such 
resources” (Bird \& Simon n.d.). Information from OLAC was used to determine language endangerment (e.g. speaker numbers) and the number and kinds of resources available in other archives for each language in PARADISEC. PARADISEC itself was used as the source of language locations, language names, and audio data.

The visualisation that uses data from these sources is titled 'Glossopticon'(Figure 1). When using the system, a user moves across a representation of a geographic region, such as Vanuatu, or Papua New Guinea and is surrounded by spatialised audio of the nearby languages. The location of each language is represented by a pillar of light surrounded by a semi-transparent dome the size of which corresponds to the approximate number of speakers. A HUD² overlay can be switched on and off, which shows the amount and diversity of archival materials (grammar, lexicon, multimedia, etc) and a documentation score for the language at which the user is gazing. In one experimental version of this system, when the user gazes at a language for long enough, the language is 'bookmarked' and a link back to its entry in the archives is added to a personal webpage generated for the user to visit after they exit the VR experience. More detail of the technical implementation of the visualisation can be found in Burrell, Hendery \& Thieberger (2019). Such a visualisation could be adapted to represent the holdings of other kinds of archives, so the discussion of it in this paper has implications for many areas of the digital humanities and research through design.

A HUD, or heads up display, is a 2D overlay of information displayed in front of the viewer's near depth of view, attached their relative position in space, as opposed to being located at a fixed position in the virtual space itself. 


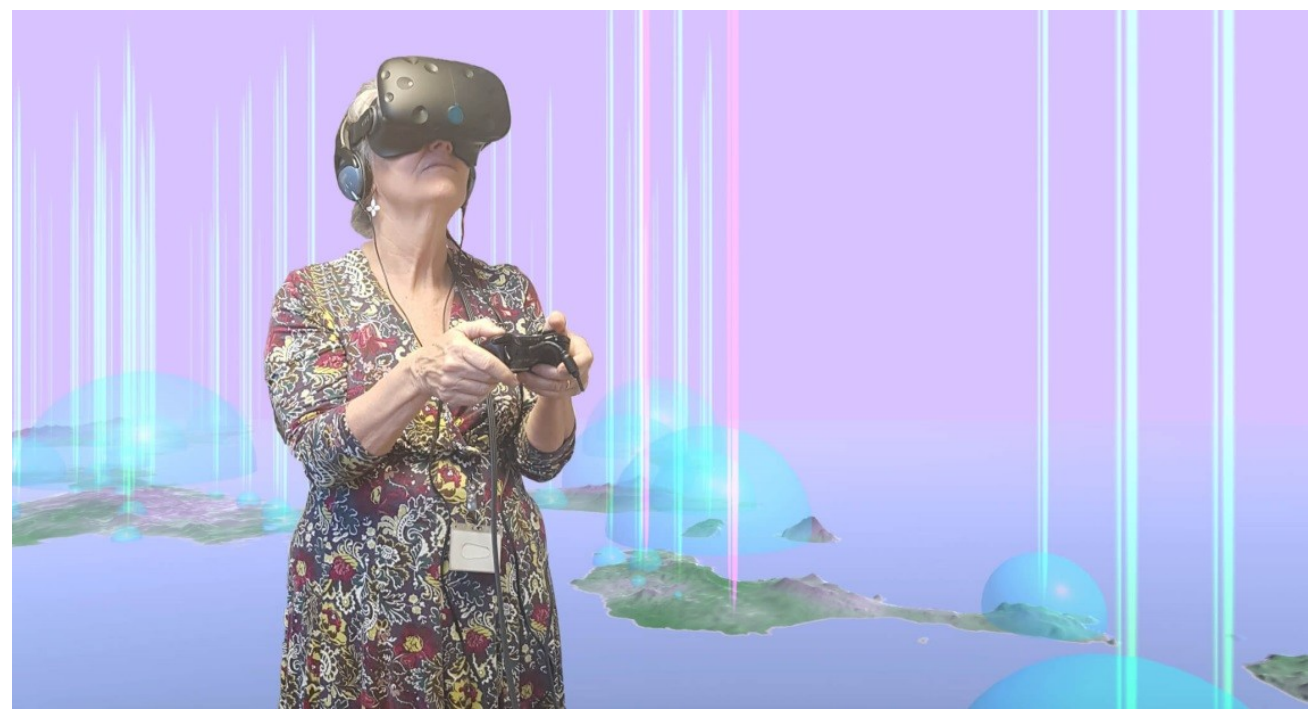

Figure 1. Composited image of Dale Middleby, curator at Canberra Museum and Gallery, exploring the Glossopticon visualisation. Image by Andrew Burrell

Subsequently, lessons learnt through experimenting with more abstract and flexible approaches to representing data have informed new, experimental versions of the Glossopticon visualisation. These visualisations, while true to the 'mapping data to landscape' modality of the original, abstract the data somewhat further, with high level information about the languages represented, such as location (via a centroid), spread (via a rectangular region) and number of speakers, represented in relation to each other, rather than directly mapped to a cartesian landscape.

The second visualisation to be discussed here is much more abstract and even more adaptable to different kinds of data. It is simply a three-dimensional cloud of coloured points laid out on three axes in space. It will be referred to it in the rest of this paper as the '3D scatterplot visualisation'. The user is located initially in the midst of this cloud, and can move through it in any direction. The colours, sizes and shapes of the points, as well as their location on the $\mathrm{x}, \mathrm{y}$, and $\mathrm{z}$ axes, can be mapped to any variables of any kind of data, and therefore this visualisation too has implications beyond linguistic research, but for the purpose of this visualisation, they 
represent linguistic features derived from the World Phonotactic Database (Donohue et al., 2013) which includes the variables from the World Atlas of Language Structures (WALS) (Dryer and Haspelmath, 2013), but also a number of others, and has an expanded sample of languages. These databases contain information on more than 2500 languages, coded for features such as number of vowels, number of consonants, word orders, how they form words, whether they have particular terms for certain grammatical functions, and references to scholarly literature about the languages. These languages and their features are archived in the databases in question by members of the linguistics community, and are subject to careful moderation by the editors. The databases are primarily used by linguistic typologists to compare and map languages.

Hendery used multidimensional scaling (Shepard, 1962a, b) to find a 3-dimensional solution in which the distances between the languages in the World Phonotactics Database scale according to how similar the languages are to each other on the 257 variables (see Hendery \& Kalyan 2016). Colouring the points in the cloud according to the geographical location of the corresponding languages or by their historical relationships results in a visualisation that makes it easy to explore clusters of languages that are structurally similar and how these similarities relate to a shared history versus proximity.

This current paper concentrates on the visualisation itself rather than the linguistic analysis. This visualisation was initially programmed in WebGL for use in-browser or with a mobile-phone-based lower-end VR headset, but recently Hendery also developed a version created with the Unity Game Engine for use with higher end VR headsets. 
Both of the visualisations described above can be viewed in a pseudo-3D environment using software authored in Unity or via a desktop browser, as shown for the data graph visualisation in Figure 2.

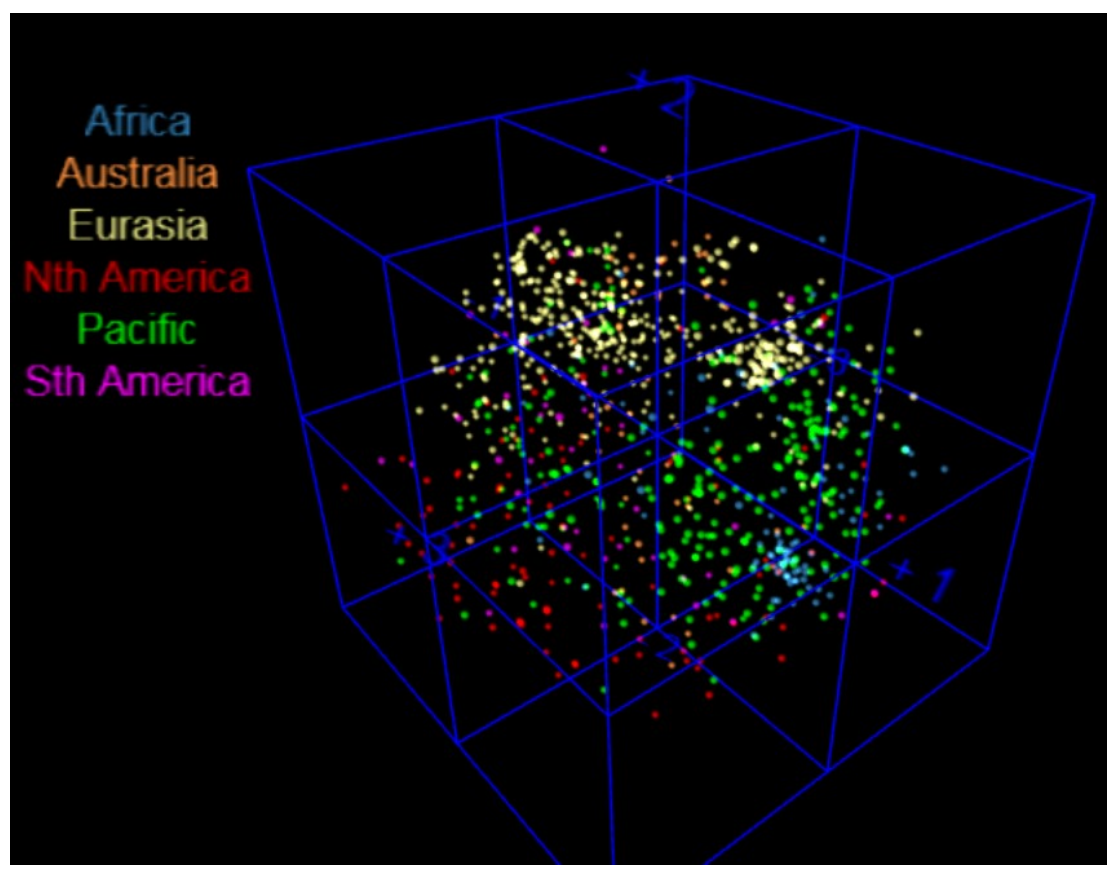

Figure 2. Screenshot of pseudo-3D data graph visualisation displayed in browser

For a more immersive VR experience, however, there are several options. First, they can be viewed inside a VR headset such as the Google Cardboard, Oculus Rift or HTC Vive(compare Figure 3). 


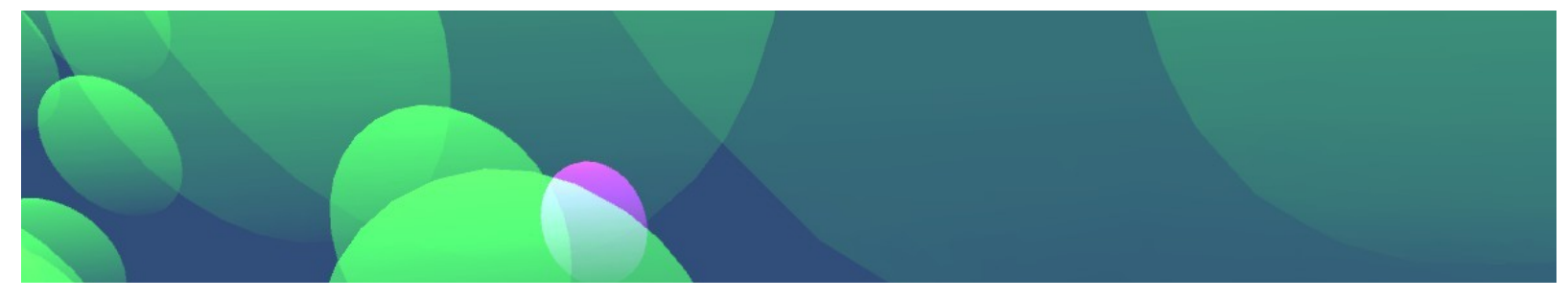

Secondly, the visualisations can be displayed on the inside of a dome such as those used in a planetarium. Through a successful LIEF grant, Western Sydney University and a number of other partners (University of Canberra, RMIT, UNSW, The National Museum of Australia, the Museum of Victoria, among others) have been able to build and host an ultra-high resolution experimental fulldome ('DomeLab’ http://www.niea.unsw.edu.au/research/projects/domelab). 
This dome provides a platform for 3D immersive visualisations of research projects using $4 \mathrm{k}$ fisheye video or interactive animation. Finally, this project has also experimented with exploring these same data environments from within the University of Technology Sydney Data Arena, a cylindrical CAVE environment.

The following sections will discuss the affordances of these different immersive technologies for innovative archival interfaces, along with some implications for humanities, research through design, and social sciences research in general.

\section{Different degrees of immersion}

Display of these visualisations via a VR headset such as the Cardboard or HTC Vive is the most straightforward sort of immersion. The viewer is embodied in the midst of the data, and simple movements from everyday life (looking around, moving forward or backward) translate directly into the virtual space. There is no direction of gaze or movement that takes the user out of the visualisation. The movement itself is currently controlled via direction of gaze (on the Cardboard), controller buttons (on the Oculus) or a combination of actual walking for small movements and 'teleporting' or simulating flight via a controller or hand gesture (on the HTC Vive) for large movements.

Display of the visualisation via a desktop web browser in software authored in the Unity game engine or WebGL, on the other hand, is the least immersive option. It nevertheless uses a translation of three-dimensional space onto a flat screen that many users are familiar with from modern computer games, and therefore can still give a sense of movement, rotation, and interactivity. The user must, however, look continuously at the screen, ignoring everything 
outside of that square, and must continuously mentally map the 2D images viewed into an imagined 3D space.

A hybrid of these two experiences which is perhaps unfamiliar to most users is a fulldome(Figure 4).

\section{Figure 4. The DomeLab fulldome as set up at UNSW.}

The user stands, sits, or lies on the floor inside the dome, and the visualisation is displayed on the inside of the curved dome over the user's head. Readers are perhaps most likely to be familiar with this kind of experience from commercial planetariums. Because the user is surrounded by the screen on most sides, the experience is far more immersive than the display of a visualisation in a computer browser, and this illusion can be extended by use of a mirror on the floor, by angling the viewer so that they are lying and gazing upwards at the dome surface, or by use of surround sound audio effects. Metaphors of movement and space have to be adapted for such a display, however, as most of the display surface is above the user, rather than fully surrounding the user, as when using a VR headset.

This has some similarities to a 'cave' or data arena space. These are rooms where the walls, and optionally also ceiling and floor, consist of screens or projection surfaces, so that, as in the dome, users are surrounded by data on all (this time flat) surfaces. One example of this is the University of technology Sydney Data Arena (Figure 9).

For the Glossopticon visualisation, a reasonable adaptation for a dome can be achieved just by angling the 'camera' downwards a little so that the horizon is higher up the dome and more space is devoted to the ground level. The data graph visualisation, on the other hand, does 
not need adaptation. While users can no longer look down to see the points on the graph that are 'below' them, this is barely noticeable, because few users inside the dome feel the instinct to look at the floor.

Within a dome or cave, users can select directions of movement on the fly, thanks to the simple inclusion of a game controller (joystick) as an interface or with more complex and experimental user interfaces. This leads to the visualisation become more interactive, but in turn hands over control of the space to one viewer on behalf of the viewers as a whole. The integration of infrared technology (such as the IR motion capture system embedded within the UTS data arena) or cameras to detect user movement are also an option that can be considered and allow for the design of more inclusive interaction schema.

We next turn to the question of why one might want to explore archival data visually, and what one gains or loses by doing so in the immersive environments described above. This discussion centres around four themes: dissemination; structure; spatialisation; and the locus of knowledge production.

\section{Considerations for immersive interfaces}

\subsection{Dissemination}

Data visualisations can have a variety of purposes. They can be a way to explore data in different configurations, in the hope of generating ideas, hypotheses, or noticing new relationships that might trigger creative ideas for future research. They can be an attempt at scientific modelling, which usually implies the testing or instantiation of more fully formed existing ideas. Or they can be a way of disseminating and communicating information to one’s peers or the general public. An early study of user experience in 2D, 3D and immersive data 
visualisation (Modjeska, 2000) showed that users generally find 2D, less immersive representations of the data more efficient. However, their enjoyment and motivation increases with the degree of immersiveness. This suggests that immersive VR data visualisations might be best suited for the playful exploration of data described above than for more serious scientific modelling. And they may be well suited for communication of ideas when it matters less whether the audience grasps the complex details than a general sense of enthusiasm and interest. This study was however, conducted on desktop, using an early web based interactive 3d graphics system³ ${ }^{3}$, where “[s]pecial input/output devices were not needed.” (Modjeska, 2000, p66).

These were therefore the initial motivations behind the two linguistic data visualisations described here. The Glossopticon visualisation was aimed at people who might like to explore what is contained in the PARADISEC archive. It is not essential that a user come away with perfect recall of what languages are located where, and how much of what type of material is in the archive for each, but rather with a general sense of the scope of the archive, and with increased interest and motivation for using the archive in the future. As Nick Thieberger, the Director of PARADISEC points out, "We've got this fantastic resource - a database of a thousand endangered languages, but it's not very engaging, it's a bit dull, so we wanted to do something to change that. We really wanted to look at how we could make this database more exciting for people and to get them engaging with it.” (Higginbotham, 2017). The data graph visualisation, on the other hand, is aimed more at researchers who want to creatively explore language data in order to generate new ideas, which they can follow up using other research

VRML, or Virtual Reality Markup Language. 
methods. This is the kind of 'work' that Ramsay (2014) calls the 'hermeneutics of screwing around'.

Preliminary user experience research on the Glossopticon visualisation suggests that this users do, in fact, engage with it in this intended spirit of playful exploration. Post-use survey of 21 users was carried out in which they were asked a number of questions. In one question they were asked to describe their experience in one word (freely chosen, not from a pre-generated list). The word 'fun' was used by two participants. Several others used words associated with play, such as 'experimental’, ‘exploratory’, 'novel’, ‘unaccustomed’, while still more selected words that suggested the aesthetics were central to their experience ('pink', 'hot', 'beautiful'). Other users chose words that suggested they learned something from the experience ('enlightening' (three users), 'interesting' (two users), 'insightful' (one user)). This is further supported by the fact that 15 of the 21 participants ranked their increase in understanding about languages of the Pacific after experiencing the visualisation as 3 or above on a 1-5 scale, where 5 was the highest. The word selections and the answer to this further question suggest that users experienced the visualisation as something between art, play and education. Furthermore, the majority of users ranked the essentialness of the VR platform to the experience (as opposed to seeing the visualisation on a flat screen or printed page) as 4 on a scale of 1 ('not at all important' to 5 ('it was integral to the experience'), suggesting that the immersiveness of the experience had a great deal to do with these outcomes.

\subsection{Structure}

Even this kind of creative, playful exploration, however, will only suggest new ideas if the visualisation displays important features of the data or relationships between data points that 
are novel or non-trivial. This will often be the case precisely because the three dimensional space reveals different underlying structures from those that are apparent in flat visualisations.

Lev Manovich notes that information visualisation is the representation of datasets in such a way as to reveal structure (Manovich, 2011). Linguists are obsessed with structure. Since Pāṇini’s mathematical approach to Sanskrit grammar more than two thousand years ago (see Vasu, 1891), linguists have been conceiving languages themselves as highly structured. Since the 19th century Neogrammarians if not before, 'language' as an abstraction that changes and exists outside of its speakers has also been considered a highly structured object of study. Sociolinguists are interested in the structure of speech communities and speaker networks; psycholinguists and neurolinguists in the linguistic structures of the mind and the brain. Linguistics is therefore a field that is primed to search for and find structure in its data, and is constantly searching for new ways to do so.

Within the digital humanities more generally, the idea of a linguistic visualisation most commonly conjures up the image of a text cloud, or other ways of visualizing written corpora. While some linguists work with these sorts of visualisations, a very active branch of linguistics involved in data visualisation is much less well-known in digital humanities, namely linguistic typology. This is the branch of linguistics that concerns itself with how languages are distributed within the space of all conceivable languages: i.e. what linguistic features are found where in the world's languages; which are found commonly, rarely, or not at all? Linguistic typologists most frequently display their datasets in tabular form, or on two dimensional world maps, such as those in the above-mentioned World Atlas of Language Structures (WALS). The limitations of 
2D maps, graphs and tables are partly practical ones: the limitations of what can be fit into a flat page, and limitations on what the user can make sense of in this form. Too many symbols crammed together make a map or graph unreadable. The third dimension of VR, or the pseudo third dimension of a zoomable interactive browser visualisation or wrapped image on a dome allows more and different possibilities for the spatial arrangement of data.

The data graph visualisation is an example of how the third dimension, in particular in VR, reveals structure that was not so apparent on a flat screen or page.

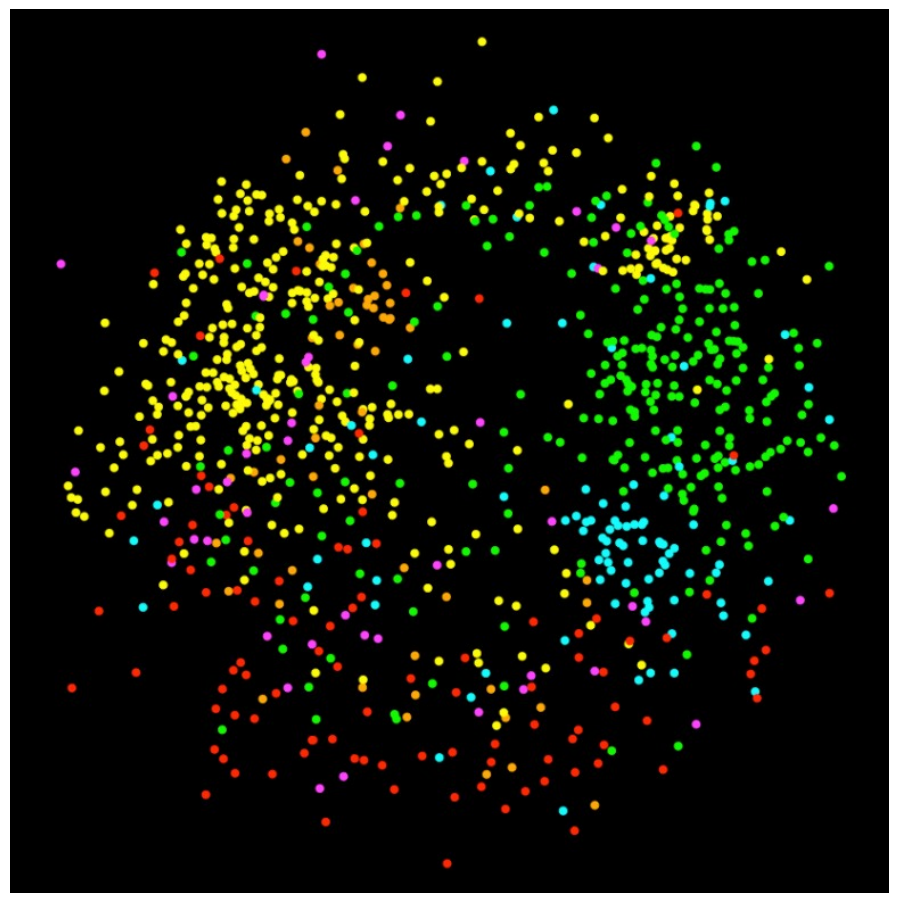

Figure 5. 2D multidimensional scaling of morphosyntactic dataset in World Phonotactics Database. Languages are coloured by region: blue = Africa; orange = Australia; yellow = Eurasia; red = North America; green = Pacific; pink = South America.

Figure 5shows a two-dimensional multidimensional scaling of the grammatical dataset derived from the World Phonotactic Database (Donohue et al, 2013). In other words, it is a 
visualisation in which each pair of languages (represented by coloured dots) is placed so that its distance represents how similar they are to each other in terms of the 257 grammatical features in the database. Languages that share grammatical features are closer together; languages that share fewer features are further apart. In this way differences and similarities along 257 parameters are reduced to an $\{\mathrm{x}, \mathrm{y}\}$ coordinate pair. This multidimensional scaling was carried out by Siva Kalyan (see Hendery \& Kalyan 2016).

It is not possible to find a solution in a lower number of dimensions that retains exactly the same relative distances for the datapoints as would exist in a 257-dimension space. The degree to which the relative distances have changed is known as 'skewing' and the amount to which a dataset is 'skewed' under multidimensional scaling can be quantified as a 'stress value'. The stress value for the morphosyntactic dataset is 27 . When the dataset is reduced to three dimensions, instead of two, the stress value is only 19, which shows that the 3D plot, unsurprisingly,more accurately represents the structure of the 257-dimension data.

The structure of the data in the Glossopticon project, similarly, is more apparent, and more immediate in three dimensional space, particularly when the user is immersed in it. While the aims of the Glossopticon project remain firmly rooted in dissemination to a wide and general audience, and a more specific dissemination of material back into the communities where the recording of the language data were made, the fact remains that the three dimensional structure of the data as visualised has provided some immediate and key insights into aspects of the linguistics behind the data - insightful for both the lay person and a linguistic researcher alike. Take for example the comparison of the spread of area a language is spoken in with the number of speakers represented in Figure 6. A significant difference is apparent immediately between 
Australian languages and those of Papua New Guinea. While this is apparent from a screen based visualisation, as represented in the figure, the actual scale of the difference is not only apparent, but felt as an immersive experience from within the environment in a VR headset. This spatial immersion, in the spatially structured data really shows what is at stake and for dissemination purposes is much more powerful.

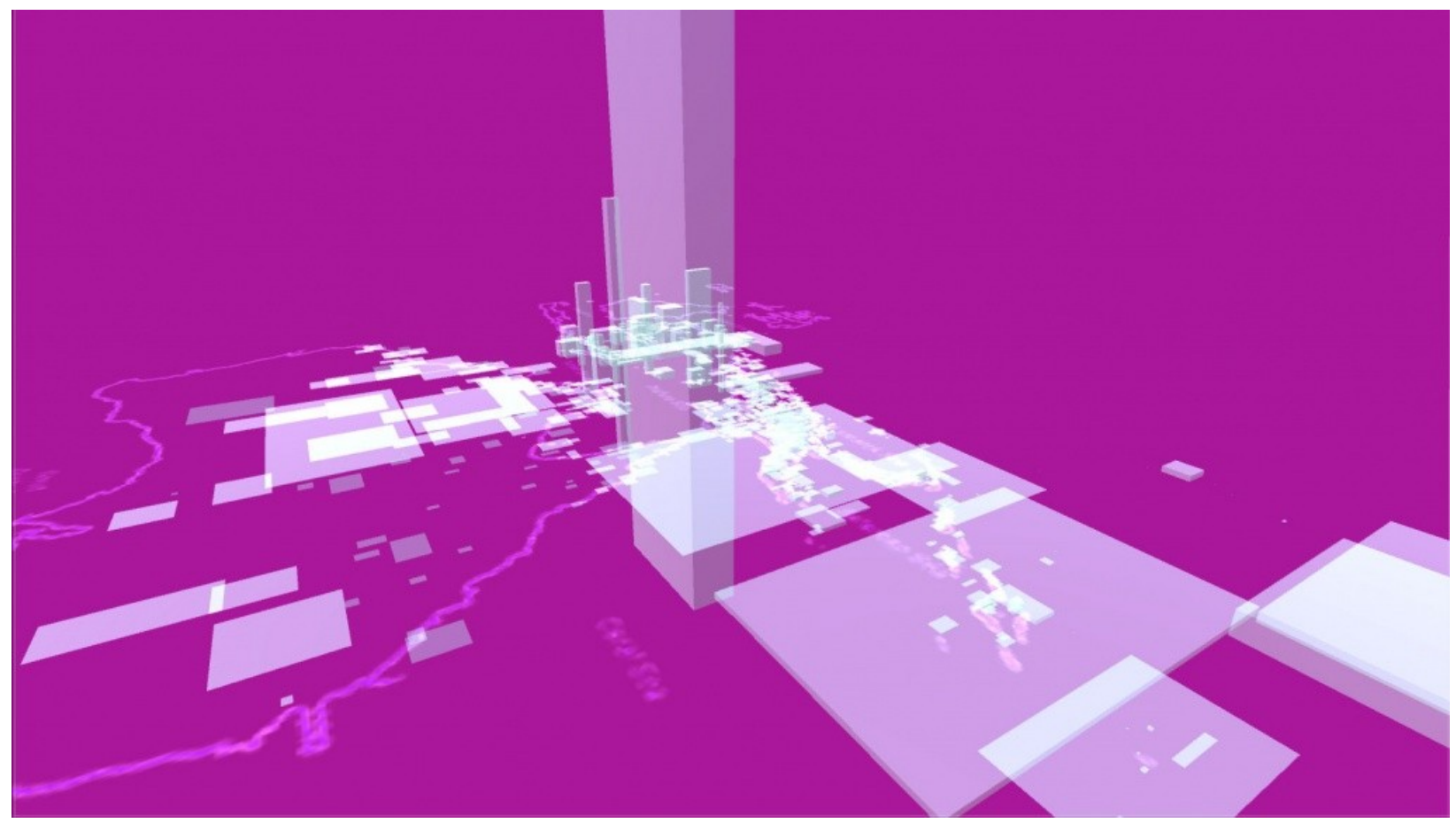

Figure 6: Languages are mapped with their spread (width and breadth) and number of speakers (height) in a recent, experimental version of the Glossopticon project.

\subsection{Spatialisation}

Manovich (2011) also claims that data visualisation is (usually) a matter of reduction and spatialisation, and that often it involves a remapping of the non-visual to the visual. There is nothing new in linguistics about reducing linguistic data to features, and remapping it to visual data (audio to text, for example). This is business as usual. What is important for the projects 
discussed here is the spatialisation of the data, and the embodiment of the researcher(s) within that data space.

Because linguistic data are already located in geographic space, the predominant linguistic visualisation among typologists is a map. It is natural to use the spatial dimensions of a page or a screen to display the spatial dimensions already inherent in the data. This is what Glossopticon does. However, Manovich (2011) also points out that spatial dimensions have a primacy in human cognition: they tend to be treated as the most important information both by visualisation creators and users. Therefore, it makes sense also to experiment with mapping them to other, perhaps more significant features of the linguistic data than the locations of the languages from which the data comes. This is the impetus behind the second, data graph visualisation. By translating the World Atlas of Language Structures data, depicted on their website on geographic maps, to a more abstract 3D graph, different relationships and structures become apparent and languages cluster in different ways.

The points representing languages can be coloured according to any schema desired. The geospatial information can be somewhat preserved by colouring each language point according to the region of the world the language is spoken in as in Figure 5. Even in this case, however, the geospatial information is secondary to the positions in space. The South American languages, for example, are scattered among the other languages, widely throughout the virtual space; the African languages on the other hand form a tight clump in their own area. This indicates that African languages are structurally more similar to each other than they are to languages from other regions of the world, while the South American languages have a wider variety of 
structures. This is less immediate on a geographical map even when similarity is coded through colour or shape (like the World Atlas of Linguistic Structures maps).

Moreover, because the researcher is embodied in the virtual space, he or she can physically move among the data points. This has some similarity to what one does when one closely examines a two-dimensional figure or map, in that the eyes might focus on a particular cluster of points, relegating others to the peripheral vision. But the virtual space allows one to do this even more literally. The user can position his or her body such that the data he or she is not interested in is behind and completely invisible, or so that a few points fill the whole field of vision. This phenomenon is a result of the spatial affordances of VRand results in an understanding of the data presented via processes of bodily cognition. Margaret Wilson points out that cognition is situated in the environment, and as a result, cognitive processes can and are offloaded into the environment. "We make the environment hold or even manipulate information for us, and we harvest that information only on a need to know basis” (Wilson, 2002, p.626). As VRevolves as a medium, it is fast becoming apparent that the same holds true of virtual environments.

While the data graph visualisation is set up to give full control to the user to move anywhere he or she desires within the space, it would be possible to pre-determine places of interest, either manually or algorithmically, and take the user on a guided tour of these parts of the dataset. This might mean, for example, drawing to their attention outliers and/or places of particular high or low density of points, and/or places where data points were unusually homogenous or unusually heterogenous in colour. This approach could be problematic: because 
most positions within the virtual space do not allow for a full view of all data points at once, much of the data set would be invisible to a researcher who relied on a tour of pre-computed points of interest. Currently, in a two-dimensional scatterplot printed on a page, an author might emphasise particular areas of the figure in some way, or point them out by description in the accompanying text, but seeing a full overview of the visualisation is still unavoidable.

\subsection{Communal knowledge production}

Finally, the possibilities opened up by the affordances of the VR headset versus other kinds of data visualisation, immersive or otherwise, have implications for the locus of knowledge production. In many humanities disciplines researchers are conceptualised as 'lone wolves', individuals who generate ideas in a relative vacuum. The important connections for the researcher are among and with the data itself, not with other people. In some disciplines, a community of research is understood to exist, but the 'conversation' among this community takes place at a remove, asynchronously, in publications, and may well include long-dead scholars. Even in this case, the primary relationship is seen as the scholar with his or her texts.

For such research, a VR headset is ideal. In the world of the headset, nothing exists but the researcher and the data: the mundane world is quite literally blacked out, and the researcher is all alone in the world the computer has created. A figure on a page, on the other hand, is agnostic about whether the viewer is a single researcher alone with his or her thoughts, or a group of scholars sitting together and discussing the paper. 
Other kinds of immersion, however, are the opposite of VR in this respect. A dome, for example, is designed to be experienced communally. The floor can be covered with large cushions or beanbags, so that people lie head-to-head, side-by-side (Figure 7). The world outside the dome is mostly blacked out, but people come and go from the dome, drawing connections to the outside world. It is natural to discuss the visualisations on the dome around you with those who lie there by your side in the half-dark. In a way, the 'research' conversations become a kind of academic pillow-talk, naturally imbued with the playfulness, intimacy, and gentleness of such. In the VR headset, you become part of the data; in the dome you become part of a community. This dichotomy starts to break down when faced with possibilities afforded by multi-user networked virtual environments, but these as yet are rare and experimental. 


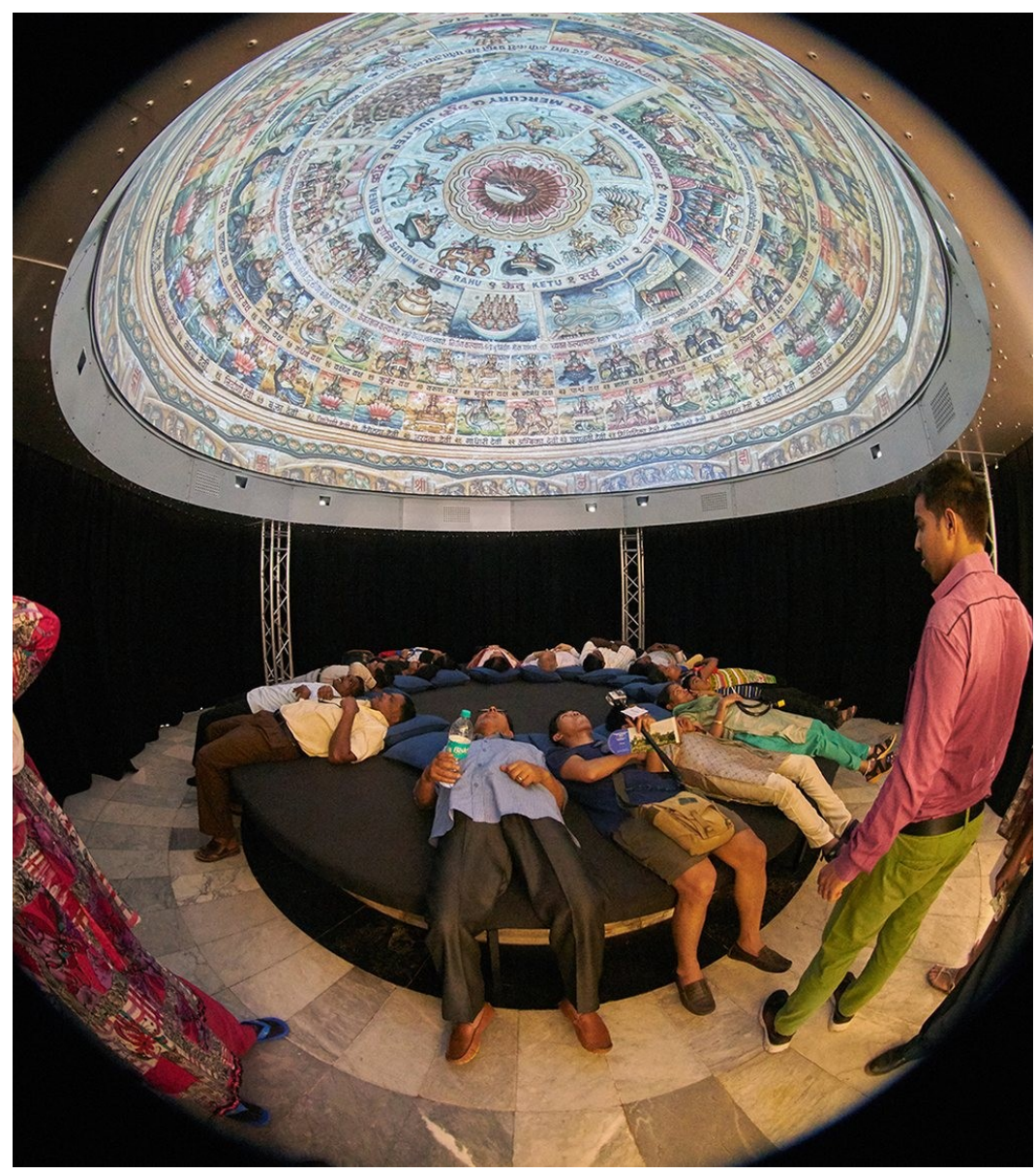

Figure 7. A communal experience of a fulldome. Look Up Mumbai, 28 January - 28 February, Mumbai, Chhatrapati Shivaji Maharaj Vastu. Sangrahalaya, Mumbai, India. Kenderdine, S, Lintermann, B and Shaw, J (2016). Image Sarah Kenderdine.

A final kind of immersive data environment is the 'cave'(Figure 8), which was introduced above. These too have potential as communal spaces for knowledge generation, but one could argue that this is a different kind of experience from that of the dome, because of the different metaphors it invites. Domes are generally known, if at all, from planetariums. These are places people visit as a special event, and they stay for a set period of time to watch a preprepared show. This expectation seems to carry over to non-astronomy domes, which are also often set up to show a timed show, sometimes with tickets, and people will visit them for the experience. The natural response is to map “attending a performance” behaviours to the dome 
experience: sitting quietly, letting attention be led by the creator of the material; not talking; staying for a limited period of time. An example of this was Lynette Wallworth’s "Coral” a full dome experience presented at the Sydney Carriageworks venue in 2017 (Wallworth, 2017), where all of these characteristics could be seen in the behaviour of the viewers as they were immersed in the work.

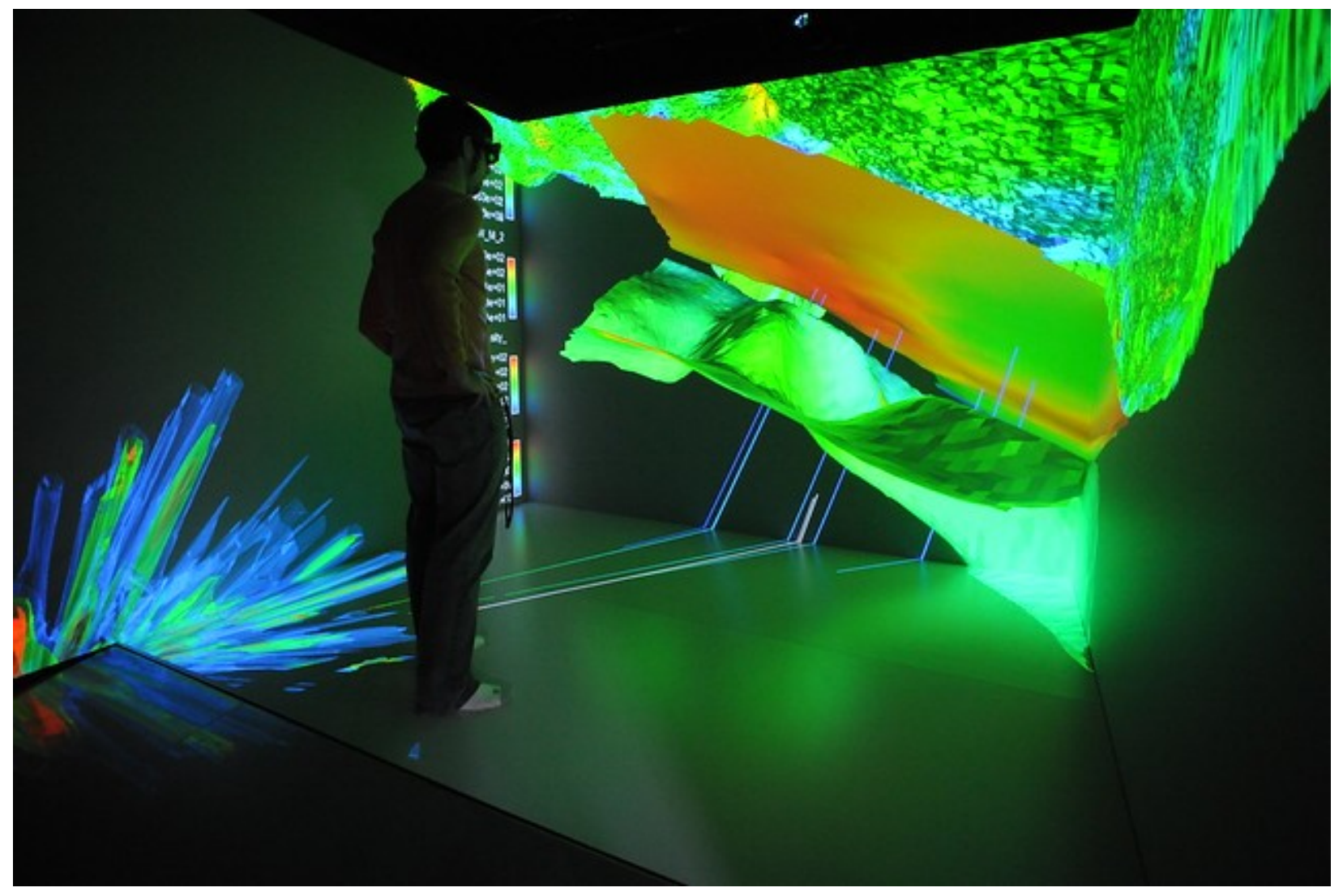

Figure 8. Computer-Assisted Virtual Environment (CAVE) at INL's Center for Advanced Energy Studies / Source: Idaho National Laboratory (2012)

A cave, on the other hand, presents the 'show' literally on the walls of the room: it is wallpaper. Wallpaper is in the background; it is unobtrusive unless one choses to notice and 
comment on it. This metaphor carries over to the virtual 'wallpaper' people put on their digital devices as backgrounds. The interaction between people in the room is what is foregrounded. One can easily imagine a situation in the future where academics might work in spaces that are set up as caves, so that relevant data can be projected in the background, and easily discussed and manipulated by colleagues meeting in the room. Libraries and archives might have walls that consist of such interactive wallpaper displaying data from their holdings. This would not be an event in the same way as the dome is, but rather an adjunct to the daily routine. A dome is a place one visits; a cave is a place one might spend a whole day. This is the sort of research model that is common in the sciences and has not, historically, been in the humanities. Archives, libraries and museums with their diverse range of visitors and complex, multi-layered holdings, could potentially benefit from the collaborative insights that arise from sitting with data and social playing.

This can be seen in the work in the UTS Data Arena while experimenting with CAVEbased outcomes for the Glossopticon project(Figure 9). The researchers work at a terminal in the center of the space, surrounded by the data environment they are designing and realizing in real time. They are constantly surrounded, in 360 degrees, by the proceeds of their work and can work in a way more conductive to projects based in collaboration. Indeed, the process of collaborating together in the data can be seen as an end in itself, and in many cases does not result in a "showing" of an outcome outside of the researchers themselves. 


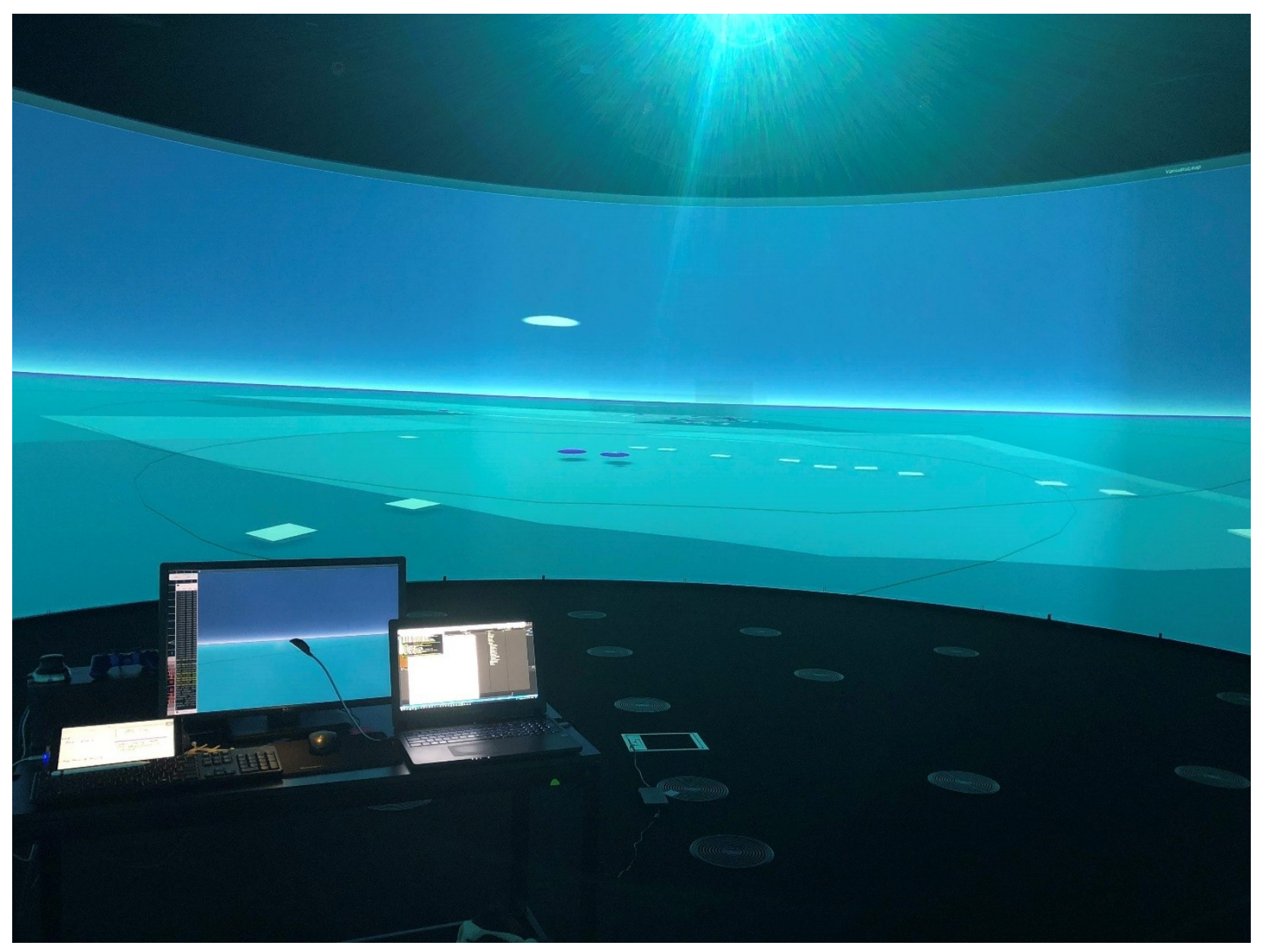

Figure 9: Demonstration of working in the UTS Data Arena

Metaphors and their associations are very important in determining how people use digital devices, and frequently are deliberately chosen to encourage the user to organise their digital practices in a particular way (for example, ‘windows', ‘files’, ‘folders', ‘desktops’ on desktop or laptop computers.) While the dome might be most easily thought of as an event, and the cave as wallpaper, a pseudo-three dimensional visualisation running in on a computer is more likely to be seen as a task with a beginning and an end, with goals, perhaps as work, or as a game, played socially or individually. It is software you use at a specific time, for a specific purpose, in a specific place. These are the expectations for most pieces of software users run on their computers in this way. 
A VR headset is part of the same class of personal electronic devices that includes laptops, tablets, smartphones. The natural behaviour is to map one’s "using an electronic device” behaviour to the VR equipment: exploring the interface; choosing a path; picking it up and putting it down as desired.Society is at a point in technology history where devices have become more social. Instead of individuals sitting separately at terminals, they pass tablets back and forth to show each other media; they play social games; household smart devices (e.g. Amazon Echo or Google Home) serve a whole group of people. VR is only just on the verge of supporting this sort of social behaviour.

Such expectations and associations are likely to have significant effects on how users interact with the very same data visualisation when it is presented in these different contexts, a hypothesis that is ripe for experimental investigation. When these users are visitors to an archive or cultural institution, there are circumstances in which it makes sense to trigger the metaphor of a "show" or "performance" . If, however, the users are researchers, a passive viewing experience of the holdings data is problematic; interactive immersive engagement, particularly in dialogue with other researchers is likely to provide more benefit in terms of productive research engagement with the archive.

\section{Conclusions}

Both of the projects discussed have experimented with new technologies that facilitate access to archival data visualised in virtual environments with an eye to audiences and accessibility. A potential implication of cultural institutions moving towards these kinds of immersive exploration and discovery interfaces in the future is that it risks making the collections more accessible to some users at the expense of others (e.g. those who do not have the access to infrastructure required) Working across the spectrum of different hardware and 
software solutions, however, has enabled these projects to experiment with possibilities of design, immersion, structure and specialisation of data, in a way that informs dissemination on more accessible technologies (eg: Google Cardboard). It is vital to be working critically and engaging in these spaces in order to bring to them the voice of the humanitarian scholar, and open up these spaces for more diverse usage.

One of the reasons the wide range of more and less immersive technologies are discussed in this paper is that there is no one ideal output solution for each project. For a project like Glossopticon that relies on data collected from diverse, sometimes remote communities, which themselves may not have access to high-end technology, it is important to produce an output that can be experienced by the community members themselves. This is one reason why these projects include web-based versions that run on mobile phones in low-cost (cardboard) VR headsets. For various other uses, a desktop-based, headset-based, or dome/cave version of the visualisation might be appropriate. In the discussion above, some affordances of each are outlined, and how they may 'read' differently to users.

This experiment with immersive archival interfaces also has implications for humanities and social sciences research more generally. One of the challenges of visualising any humanities dataset is the inherent uncertainty, subjectivity, and messiness (Posner 2015). Attractive data visualisations risk misleading users into believing that they are viewing statements of facts, or a direct representation of the real world. However, connecting one dataset and a way of visualising it to multiple different kinds of output (desktop, VR headset, dome, cave, etc) draws users' attention to the fact that the map is not the territory - any one of those visualisations is just one lens through which the data can be examined. This is even more so the case when the same underlying data (e.g. languages of the Pacific) can be viewed in two or more completely different 
visualisations: one privileging the geographical dimension of the data and one reducing that to a minor variable, in favour of the structural features of the languages.

In a library, archive, or museum setting, the various approaches to virtual interfaces could be simultaneously deployed, so that different kinds of users could experience or investigate the holdings in a way that makes sense to them, and allows them to achieve their goals, whether those be information discovery, retrieval, or even entertainment. The various options, with different levels of technological requirements, literacy, and expense, also potentially allow dissemination of archival, library or museum holdings to users in different circumstances and locations, including traditionally marginalised communities for whom traditional interfaces are not especially accessible.

\section{References}

Bates, M.J., 1977. Factors affecting subject catalog search success. Journal of the American Society for Information Science 28, 161-169. https://doi.org/10.1002/asi.4630280304

Bird, Stephen, and Gary Simons. n.d. "Open Language Archives Community." Accessed March 27, 2019. http://www. language-archives.org/.

Burrell, A., Hendery, R. and Thieberger, N. (2019), Glossopticon: visualising archival data. Proceedings of the 2019 23rd International Conference in Information Visualization. Part II, IV-2 2019: Adelaide, South Australia, 16 19 July 2019, 100-103. https://doi.org/10.1109/IV-2.209.00029

Chow, A., Baity, C., Chappel, P., Rachlin, C., Vinson, C., Zamarripa, M., 2010. When real and virtual worlds collide: a public library's management of a second life library. ALA Virtual Communities and Libraries Membership Interest Group online conference.

Davies, C., 1998. The Virtual Dimension: Architecture, Representation, and Crash Culture (1st. ed.). Princeton Architectural Press, New York, NY. Donohue, Mark, Rebecca Hetherington, James McElvenny, and Virginia Dawson. 2013. "World Phonotactics Database." 2013. http://phonotactics.anu.edu.au/.

Dryer, Matthew S., and Martin Haspelmath, eds. 2013. WALS Online. Leipzig: Max Planck Institute for Evolutionary Anthropology. https://wals.info/.

Efthimiadis, E.N., 1990. Online Searching Aids: A review of Front Ends, Gateways and Other Interfaces. Journal of Documentation 46, 218-262. https://doi.org/10.1108/eb026861

Heim, M., 1993.The Metaphysics of Virtual Reality. Oxford University Press, New York 
Hendery, R. and Kalyan, S. (2016), Within these WALS: an immersive exploration of linguistic space. Poster presented at the Second Annual COEDL Fest, Sydney, February 2016.

Higginbotham, W., 2017. Can virtual reality help save endangered Pacific languages? [WWW Document]. ABC News. URL https://Www.abc.net.au/news/2017-0729/researchers-turn-to-vr-to-save-endangered-pacific-languages/8743702 (accessed 3.27.19)

Idaho National Laboratory (2012), Data Immersion [digital image]. Retrieved from https://WwW.flickr.com/photos/inl/9196075004

Kenderdine, S, Lintermann, B, and Shaw, J. (2016), Look Up Mumbai, Look Up Mumbai, Chhatrapati Shivaji Maharaj Vastu Sangrahalaya (formerly the Prince of Wales Museum), Mumbai, Editor(s): Jindal Art Foundation, fulldome, [Recorded / Rendered Creative Works] http://www.niea.unsw.edu.au/events/domelab-lookmumbai

Lanier, J., 1989. An Interview with Jaron Lanier. Whole Earth Review 64 (FALL 1989), 8

Laurel, B., 1993. Computers as Theatre. Addison-Wesley: Reading, Mass.

Laurel, B., Strickland, R., Tow, R., 1994. Placeholder: landscape and narrative in virtual environments. ACM SIGGRAPH Computer Graphics 28 (1994), 118-126.

Lugmayr, A., Greenfeld, A., Woods, A., Joseph, P., 2016. Cultural Visualisation of a Cultural Photographic Collection in 3D Environments - Development of 'PAV 3D' (Photographic Archive Visualisation), in: Wallner, G., Kriglstein, S., Hlavacs, H., Malaka, R., Lugmayr, A., Yang, H.-S. (Eds.), Entertainment Computing - ICEC 2016. Springer International Publishing, Cham, pp. 272-277. https://doi.org/10.1007/978-3-319-46100-7 29

Manovich, L. 2011. What is Visualization?, Visual Studies, Vol. 26 No 1, pp. $36-$ 49.

McLuhan, Marshall. 1964. Understanding Media. New York: McGraw Hill.

Miller, R.A., 1942. On the use of the card catalog. The Library Quarterly 12, 29637.

Modjeska, D.K., 2000. Hierarchical data visualization in desktop virtual reality. National Library of Canada = Bibliothèque nationale du Canada, Ottawa.

Posner, M., 2015. Humanities data: a necessary contradiction? Talk at the Harvard Purdue Data Management Symposium on June 17, 2015, in Cambridge, Massachusetts. Reproduced at https://miriamposner.com/blog/humanities-data-anecessary-contradiction/

Poulter, A., 1993. Towards a virtual reality library. Aslib Proceedings 45, 11-17. https://doi.org/10.1108/eb051300

Ramsay, S., 2014. The hermeneutics of screwing around; or what you do with a million books, in: Kee, K. (Ed.), Pastplay: Teaching and Learning History with Technology. University of Michigan Press, Ann Arbor, Michigan, pp. 111-120.

Riha, D., 2010. The 3-D virtual library as a value-added library service, in: Maj, A. (Ed.), Emerging Practices in Cyberculture and Social Networking, At the Interface / Probing the Boundaries. Brill Rodopi, Amsterdam, pp. 119-134.

Shepard, Roger N. 1962a. "The Analysis of Proximities: Multidimensional Scaling with an Unknown Distance Function. I." Psychometrika 27 (3): 125-40. https://doi.org/10.1007/BF02289621.

Shepard, R.N., 1962b. The analysis of proximities: Multidimensional scaling with an unknown distance function. II. Psychometrika 27, 219-246. https://doi.org/10.1007/BF02289621

Stevens, R.E., 1950. A summary of the literature on the use made by the research worker of the university library catalog. Occasional papers (University of Illinois at Urbana-Champaign. Graduate School of Library Science) 13.

Tagliacozzo, R., Kochen, M., 1970. Information-seeking behavior of catalog users. Information Storage and Retrieval 6, 363-381. https://doi.org/10.1016/0020$\underline{0271(70) 90029-X}$ 
Thieberger, N., , Barwick, L., 2012. “Keeping Records of Language Diversity in Melanesia: The Pacific and Regional Archive for Digital Sources in Endangered Cultures (PARADISEC)." Edited by Nicholas Evans and Marian Klamer. Language Documentation \& Conservation Special Publication 5 (December): 239-53.

Turner, J., 2002. Myron Krueger Live. CTHEORY. January.

Vasu, Srisa Chandra. 1891. The Ashtadhyayi of Panini (Edited and Translated into English), Vol. I. Delhi: Motilal Banarsidass.

Wallworth, Lynette. 2017. "Coral." Full Dome Projection, The Carriageworks, Sydney, May. https://carriageworks.com.au/events/coral/.

Whitelaw, M. 2015. Generous interfaces for digital cultural collections, Digital Humanities Quarterly, Vol. 9, No. 1, available at:

http://Www.digitalhumanities.org/dhq/vol/9/1/000205/000205.html Accessed $16 / 12 / 2019$

Wilson, M., 2002. Six views of embodied cognition. Psychonomic Bulletin \& Review 9, 625-636. https://doi.org/10.3758/BF03196322 